Gergely Vadai, Zoltán Gingl and János Mellár, Real-time demonstration of the main characteristics of chaos in the motion of a real double pendulum, 2012 Eur. J. Phys. 33907 doi: $10.1088 / 0143-0807 / 33 / 4 / 907$

Final manuscript

\title{
Real-time demonstration of the main characteristics of chaos in the motion of a real double pendulum
}

\author{
Gergely Vadai ${ }^{1}$, Zoltán Gingl ${ }^{1}$ and János Mellár ${ }^{1}$ \\ ${ }^{1}$ Department of Technical Informatics, University of Szeged, Árpád tér 2, 6720 \\ Szeged, Hungary \\ e-mail: vadai.gergely@stud.u-szeged.hu
}

\begin{abstract}
Several studies came to the conclusion that chaotic phenomena are worth including in high school and undergraduate education. The double pendulum is one of the simplest systems which are chaotic, therefore, the numerical simulations and theoretical studies of it have been given large publicity, and thanks to its spectacular motion, it has become one of the most famous demonstration tools of chaos, either through simulations or in real experiments. Although several attempts have been made to use the experiment in laboratory exercises, as the friction in the real experiment changes the nature of the motion and the values of characteristic parameters during the motion, examining the measured (dissipative) motion and to compare with theoretical results raise several questions. In our review, we are presenting a measurement system which is able to analyse these questions. The system, which consists of simple yet precise data acquisition electronics, easily attainable sensors, a Bluetooth module (to the communication with the PC), and an open-source software, demonstrates on-line the main characteristics of chaos and the methods of its study and allows to analyse the dissipative motion. Further information (including downloadable software) is provided on a dedicated page, http://www.inf.u-szeged.hu/noise/Research/DoublePendulum/.
\end{abstract}

\section{Introduction}

In recent decades, the investigation of the chaotic phenomena has come to the front, and it showed that these are by no means specific and rare but rather typical processes. Moreover, the appeal of these interesting phenomena, the spectacular nature of chaotic motion and its representations may prove to be significant factors in motivating students and developing their skills with computer simulations and numerical methods. Consequently, several studies came to the conclusion that the chaotic behaviour of simple mechanical systems (e.g., spring-pendulum, double pendulum, bouncing ball on dual-slope, a magnetic pendulum, anharmonic oscillators, etc..) are worth including in high school and undergraduate education $[1,2,3,4,5]$.

The double pendulum is the simplest Hamiltonian system (with four-dimensional phase space) which is chaotic (with certain initial conditions), therefore, the numerical simulations and theoretical studies of it have been given large publicity [e.g. 6, 7, 8]. Furthermore, thanks to its spectacular motion, it has become one of the most famous demonstration tools of chaos, either through simulations or in real experiments (e.g., tandem double pendulum [9]).

The question of which chaotic parameters and how are changed by the presence of friction that is inevitable in real experiments has been gaining more and more attention. In the past few years, several attempts have been made to use quantitative analysis of the motion of real double pendulums in education $[4,10,11]$. However, the question has remained open whether the chaotic parameters of dissipative motion are suitable and easily measurable like those in other demonstrating experiments which solve the problem of energy-dissipation by energy-input (e.g., driven pendulum) $[1,2,5,12]$ ? In this study, we are trying to answer these questions with a system we have developed, which measures and displays the parameters of the motion of the real double pendulums real-time: namely, 
the angular displacements and the calculated angular velocities and sections of the two pendula. The system, which consists of simple yet precise data acquisition electronics, sensors that are easily attainable sensors and have simple measurement principles and an open-source software, demonstrates the main characteristics of chaos and the methods of its study understandably, tangibly but convincingly. Therefore, the presented work can be interesting for undergraduate students and teachers, but the mentioned problem of comparison of the conservative and dissipative cases can also be exciting for graduate and $\mathrm{PhD}$ students and even for scientists who are interested in chaos or nonlinear phenomena.

In the following paragraphs, we shall review the basic theories of the studied phenomenon, the set-up and functioning of the system and the theoretical conclusions drawn from the dissipative motion of double pendulums and argue for its educational potential as demonstration tool in high school and university education. It provides an example of the mechanical and electronic construction, data acquisition and signal processing for an undergraduate teaching laboratory and can serve as the basis of many other undergraduate projects.

\section{Chaos in the double pendulum}

In everyday life, the word 'chaotic' is commonly understood as a disorder in a many-particle system. From a scientific point of view, deterministic chaos means the irregular behaviour of simple systems that can be described in terms of a few variables.

The main properties of chaotic behaviour [1]:

- aperiodic, shows irregularity in time,

- shows sensitive dependence to initial conditions, unpredictable in the long term,

- complex, but ordered in phase space; shows a fractal structure.

These properties usually imply each other.

The description of chaotic dynamics requires new methods which depend on the system under study: the treatment of conservative (frictionless) cases differs from that of dissipative (frictional) ones. Furthermore, in both cases, we distinguish by time development: the permanent and transient type of chaos. The permanent type is steady in time, whilst the transient type is temporary or steady at only exceptional initial conditions.

The necessary conditions of chaotic behaviour [1]:

- the system is non-linear,

- the movement of the system is described by at least three independent, first-order autonomous differential equations.

The observations show that the chaos is a general property of the low-dimensional systems, which makes the examination of these systems a significant part of modern physics.

It follows that the simplest Hamiltonian system (system without energy input) which shows chaotic behaviour has a 4 dimensional phase space. The double pendulum is a non-linear system of this kind (see Figure 1), so it has become a preferred subject of the examination of chaotic dynamics, and its movement has become a basic example of chaotic motion.

The kinetic energy of the system comes from the sum of the rotational and translational energy of the pendula, where the frames of reference are the centres of masses (see Figure 1):

Where

$$
T=\frac{1}{2} m_{1} \cdot \vec{v}_{1}^{2}+\frac{1}{2} m_{2} \cdot \vec{v}_{2}^{2}+\frac{1}{2} I_{1} \cdot \dot{\Theta}_{1}^{2}+\frac{1}{2} I_{2} \cdot \dot{\Theta}_{2}^{2}
$$




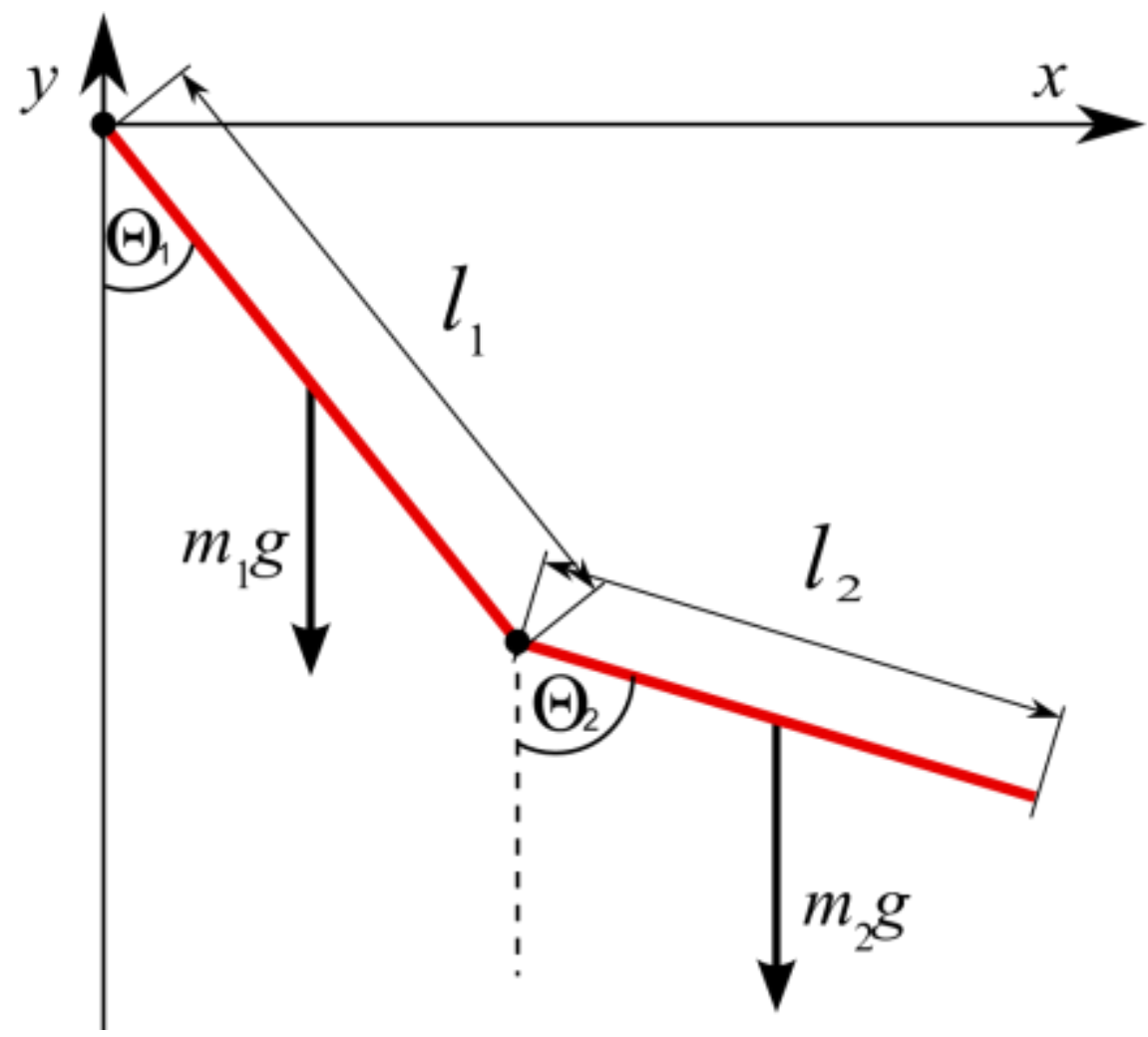

Figure 1. Schematic view of the double pendulum.

$$
I_{i}=\frac{1}{12} m_{1} \cdot l_{i}^{2}
$$

the moment of inertia for the ith pendulum,

$$
v_{i}^{2}=\dot{x}_{i}^{2}+\dot{y}_{i}^{2}
$$

where the coordinates are:

$$
\begin{gathered}
x_{1}=\frac{l_{1}}{2} \sin \Theta_{1}, \quad x_{2}=l_{1} \sin \Theta_{1}+\frac{l_{2}}{2} \sin \Theta_{2} \\
y_{1}=-\left(\frac{l_{1}}{2} \cos \Theta_{1}\right), \quad y_{2}=-\left(l_{1} \cos \Theta_{1}+\frac{l_{2}}{2} \cos \Theta_{2}\right)
\end{gathered}
$$

The potential energy of the system:

$$
V=m_{1} \cdot g \cdot y_{1}+m_{2} \cdot g \cdot y_{2}
$$

Where $g$ is the gravitational acceleration and the $\mathrm{x}$-axis is the zero level $\left(\theta_{l}= \pm 90^{\circ}\right)$.

Then, from (2.1) and (2.6) we can obtain the Lagrangian and the Hamiltonian and derive the equation of motion. The numerical solution of similar systems of differential equations has an extensive literature, calculating with a massless rod ending in a massive bob [7, 8]. To compare the solutions with real measurements, we should calculate with compound pendulums, as the equations show above (as used for example in Ref [10]).

To analyze the dissipative motion of a double pendulum numerically, quantitative estimation of friction should be made, which is rather complicated, because different types of friction appears during the motion. The differential equations containing dissipative components, estimations and measurements of effect of the friction are given in Refs [10,18].

In a conservative case, a special section of the (now 4 dimensional) phase space shows a characteristic pattern (Poincaré-section). We can describe the main properties of chaos with three characteristic numbers, namely: the topological entropy (defining the irregularity), fractal dimension (defining the phase space order), and the Lyapunov exponent (defining the unpredictability). 
A tiny separation between nearby initial conditions $\left(\Delta x\left(t_{0}\right)\right)$ are amplified exponentially in time; the exponent of this growth is called local Lyapunov exponent ( $\lambda$ a positive number):

$$
\Delta x(t)=\Delta x\left(t_{0}\right) \cdot e^{\lambda \cdot t}
$$

This characteristic number is the most easily measurable parameter in real experiments, because we can describe the nature of the motion (with given initial conditions) by calculating the separation of two trajectories which started from nearly identical conditions. The other two numbers (which are not completely independent from the Lyapunov exponent in dissipative case [1]) describe the structure of the phase space, and in contrast to numerical simulations, during the experiment it is not possible to gather enough data to perform the required examination.

\section{Measurement system}

The double pendulum (shown in Figure 2) has been constructed following the plans of Shinbrot et al. [9]. Parameters are presented in Table 1. The two aluminium pendula rotate with ball-bearings with the stand mounted on the table. To approach the ideal case, the upper pendulum consists of two flat pieces, with the lower pendulum suspended between them.

Table 1. Parameters of the double pendulum examined in the present work

\begin{tabular}{ll}
\hline \multicolumn{1}{c}{ Values } & \multicolumn{1}{c}{ Parts of the double pendulum } \\
\hline $0,273 \mathrm{~m}$ & upper pendulum length $\left(\mathrm{l}_{1}\right)$ \\
$0,215 \mathrm{~m}$ & lower pendulum length $\left(\mathrm{l}_{2}\right)$ \\
$0,025 \mathrm{~m}$ & width of the two pendula $\left(\mathrm{w}_{1}, \mathrm{w}_{2}\right)$ \\
$0,01 \mathrm{~m}$ & thickness of one half of the upper pendulum $\left(\mathrm{z}_{1}\right)$ \\
$0,02 \mathrm{~m}$ & thickness of the lower pendulum $\left(\mathrm{z}_{2}\right)$ \\
$0,369 \mathrm{~kg}$ & upper pendulum weight $\left(\mathrm{m}_{1}\right)$ \\
$0,29 \mathrm{~kg}$ & lower pendulum weight $\left(\mathrm{m}_{2}\right)$
\end{tabular}



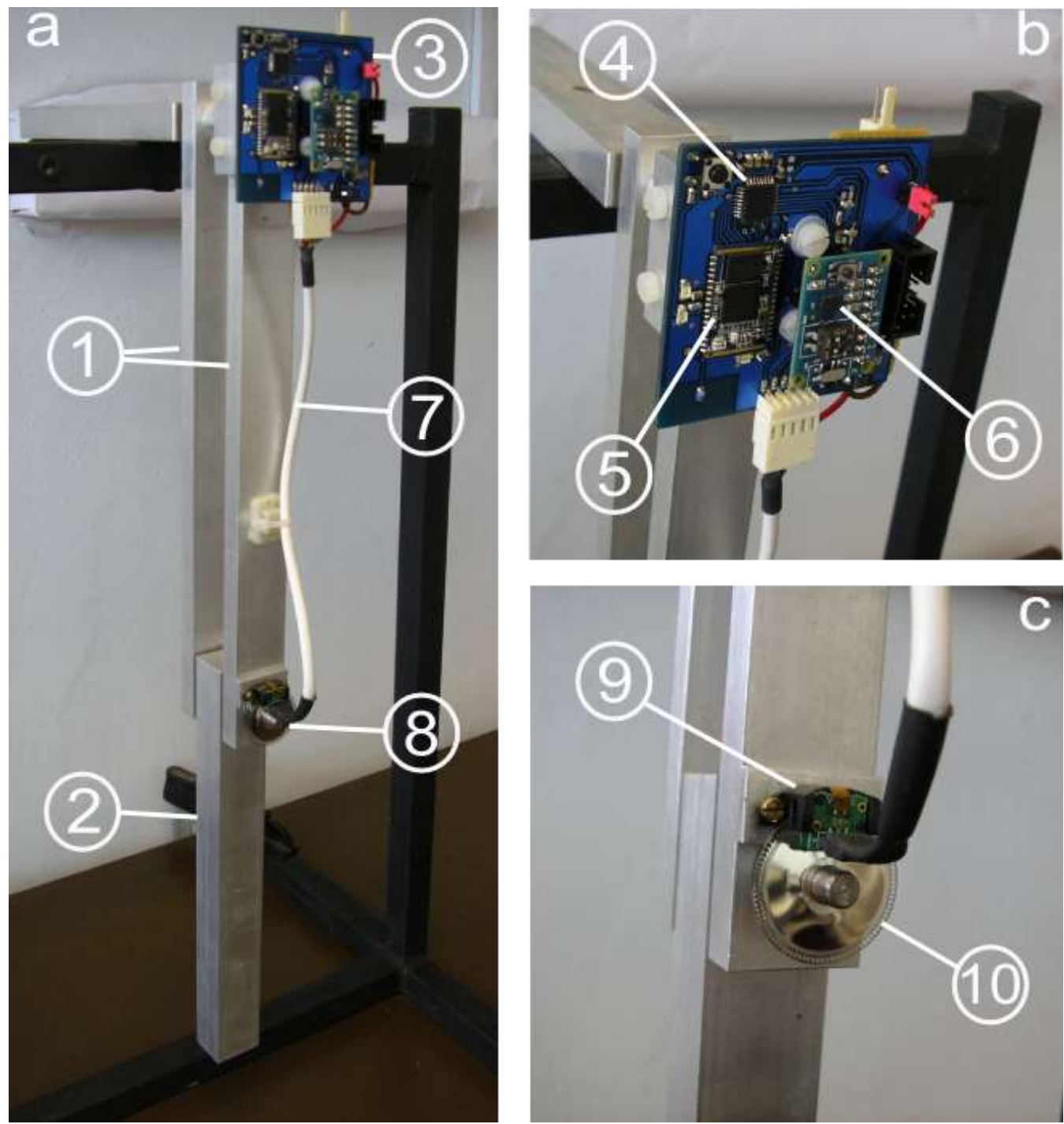

Figure 2. The main parts of the examined double pendulum: upper pendulum (1), lower pendulum (2), circuit board (3) containing the microcontroller to digitise the signals of the sensors (4), Bluetooth module (5) to the communicate wirelessly with the PC and the 3-axis accelerometer (6) to measure the angular displacement of the upper pendulum; cable (7) to bring the signal of the optical encoder to the microcontroller, optical incremental encoder (8) to measure the angular displacement of the lower pendulum and the parts of it are the light emitter and the detector unit (9) and the codewheel (10).

For the purposes of developing a measurement system to analyse the motion of a double pendulum, Ref [11] offers a detailed documentation. However, based on virtual instrumentation, we propose a more flexible and easily usable system to examine the motion of a double pendulum, as shown hereinafter.

The rotation of the two pendula is measured by an accelerometer and an optical encoder, and their signals are digitised by the analogue-digital converter of a microcontroller (shown in Figure 3). 


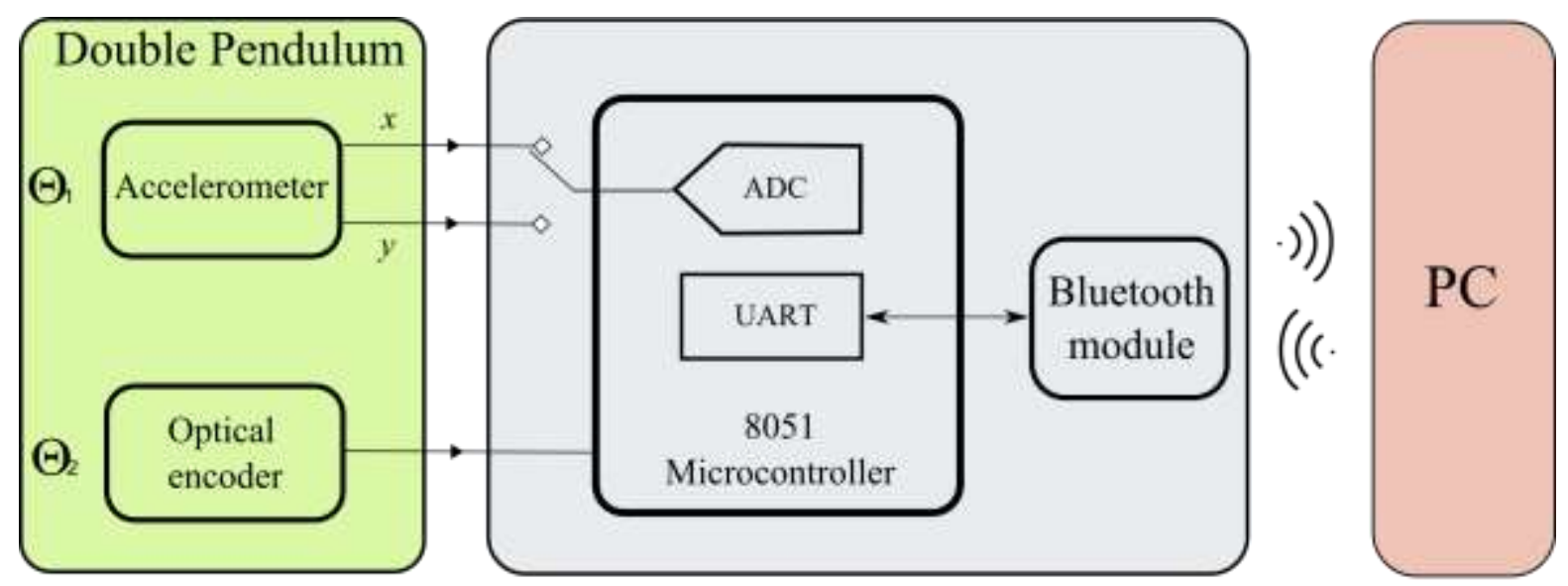

Figure 3. The block diagram of the measurement system. The sensors are placed on the double pendulum. A Bluetooth module controls the communication between the microcontroller and the PC, where the software, the 'virtual instrument' is running. $\Theta_{l}$ and $\theta_{2}$ are the angular displacements of the upper and lower pendula.

The rotation of the upper pendulum is measured by an MMA7360 3-axis accelerometer [13, 14]. Accelerometers can be used to measure tilt, since they sense the gravitational acceleration. This means, that two accelerometer channels are needed to determine the angle between the upper pendulum beam and the gravity direction in the whole range $\left(0^{\circ}-360^{\circ}\right)$ of the rotation: on channel is parallel and one is perpendicular to the pendulum rod. In such arrangement the two acceleration signal give the cosine and sine of the angle, respectively, therefore the angle can be easily calculated. This way an interesting analogue method is demonstrated, that is cost effective, easy-to-mount and much less sensitive than a codewheel. However, the centre of accelerometer should be placed at the rotation axis of the pendulum to sense the rotation only, therefore this method can't be used to measure the angle of the lower pendulum, whose the rotation axis is moving around a circle.

To measure the rotation of the lower pendulum irrespectively of the position and velocity of the upper pendulum, an AEDB 9140 optical incremental encoder was used. A codewheel (a wheel with clean and opaque areas on its margin) rotates between a light emitter unit (lensed LED source) and a photodetector unit, and interrupts the light beam depending on the current position. The microcontroller reads the two state signals generated by the detector unit and, using a codewheel with 500 space-bar pairs, can calculate the angular displacement with a resolution of $0.36^{\circ}$ (see measurement principle in [15]). The sensor gives an index signal after it rotates a whole round, so we can calibrate the actual angle at the first index signal, allowing the absolute measurement of angular displacement.

If the codewheel of the sensor is placed to the lower rotation axle and the encoder is placed to the bottom of the upper pendulum (shown in Figure 2c), the rotation becomes measurable, and this method is non-contact too

The different principles of the two rotation measurements demonstrate well the advantages and disadvantages of using analogue and digital signals. This shows that generally there are several different solutions for a problem, and it is difficult or impossible to choose the 'best' one, because of the many other conditions affecting the system. Thus it is didactically useful to demonstrate and show the different solutions and methods.

After A/D conversion, the signals of the sensors were sent to the PC with a wireless Bluetooth module [16] to avoid problems of using cables to that would affect on the motion and would not allow multiple turnarounds. The sampling frequency between the PC and the electronics was $100 \mathrm{~Hz}$ and between the electronics and sensors was $20 \mathrm{kHz}$, which is enough for detecting fast movements of the lower pendulum that produce 4-5 flips in a second.

The real-time software, which displays and analyses the movement of the double pendulum, and the subroutines of communication were written in LabVIEW. After setting the sampling frequency, the program allows the on-line monitoring of the motion. 


\section{Online measurement}

\section{1. $\quad$ Measuring angular displacement and angular velocity}

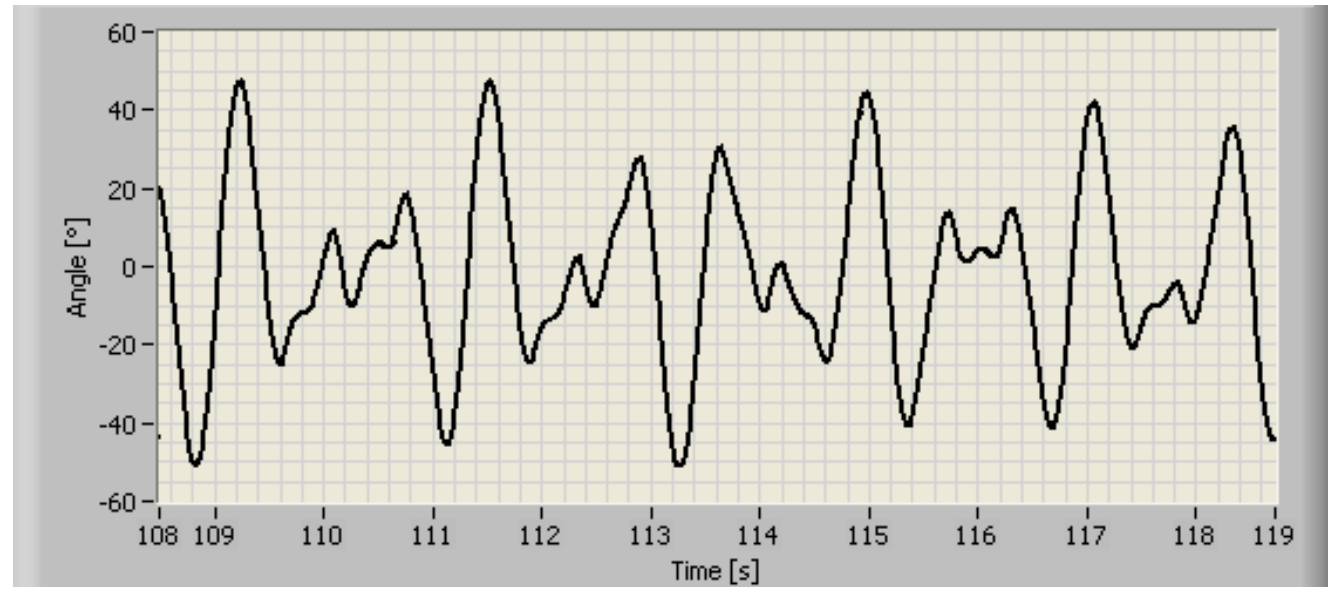

Figure 4. Online display of the angular displacement of the upper pendulum $\left(\theta_{1}\right)$.

The measurement software calculates and displays real-time the angular displacements of the pendula from the signal of the sensor (shown in Figures 4 and 5). In order to enhance the accuracy, a secondorder Bessel low pass filter was used, which in this case is optimal to cut the noise in high frequencies. Thus the two angular displacements $\left(\theta_{1}, \theta_{2}\right)$ were removed with the $5 \mathrm{~Hz}$ cut-off frequency determined from the unfiltered signal spectra. The angular velocities were calculated with numerical derivation.

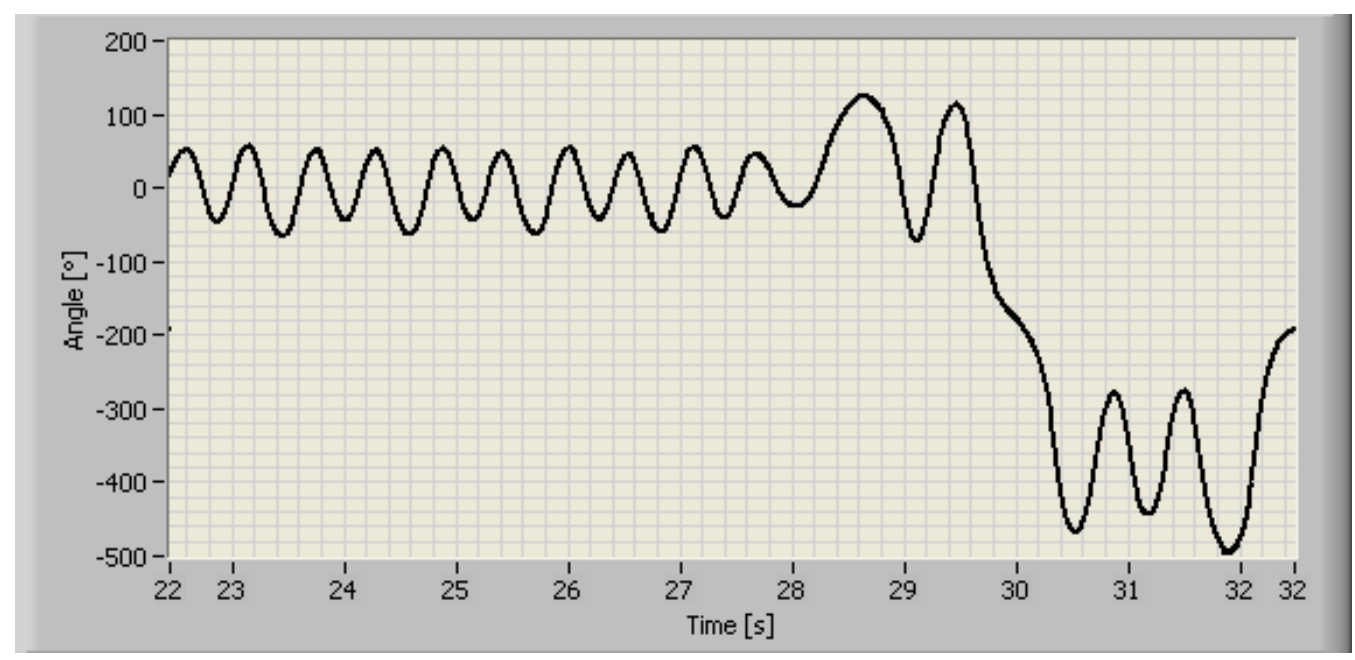

Figure 5. Online display of the angular displacement of the lower pendulum $\left(\theta_{2}\right)$.

\subsection{Demonstrating the sensitive dependence to initial conditions}

The software is able to demonstrate on-line the appearance of chaos in the experiments convincingly, to show why we call the motion of the double pendulum chaotic besides being very spectacular. One of the main properties of chaotic motion is the sensitive dependence to initial conditions. Thus, recording several paths that started with nearly identical initial conditions, and plotting them with different colours, the separation of the trajectories after the first unstable state demonstrate well the chaotic property mentioned above (shown in Figures 6). The angular displacements of the lower pendulum were untruncated (no modulo $2 \pi$ was taken), so trajectories could be shown continuously, with the big differences marking well that the number of flips of the lower pendulum flips are dissimilar, so the movements differ significantly.

It is certainly substantial to try to reach more identical initial conditions and to synchronise the launch of the movements. The accuracy required to calculate the Lyapunov-exponent could be corrected offline (see below). 


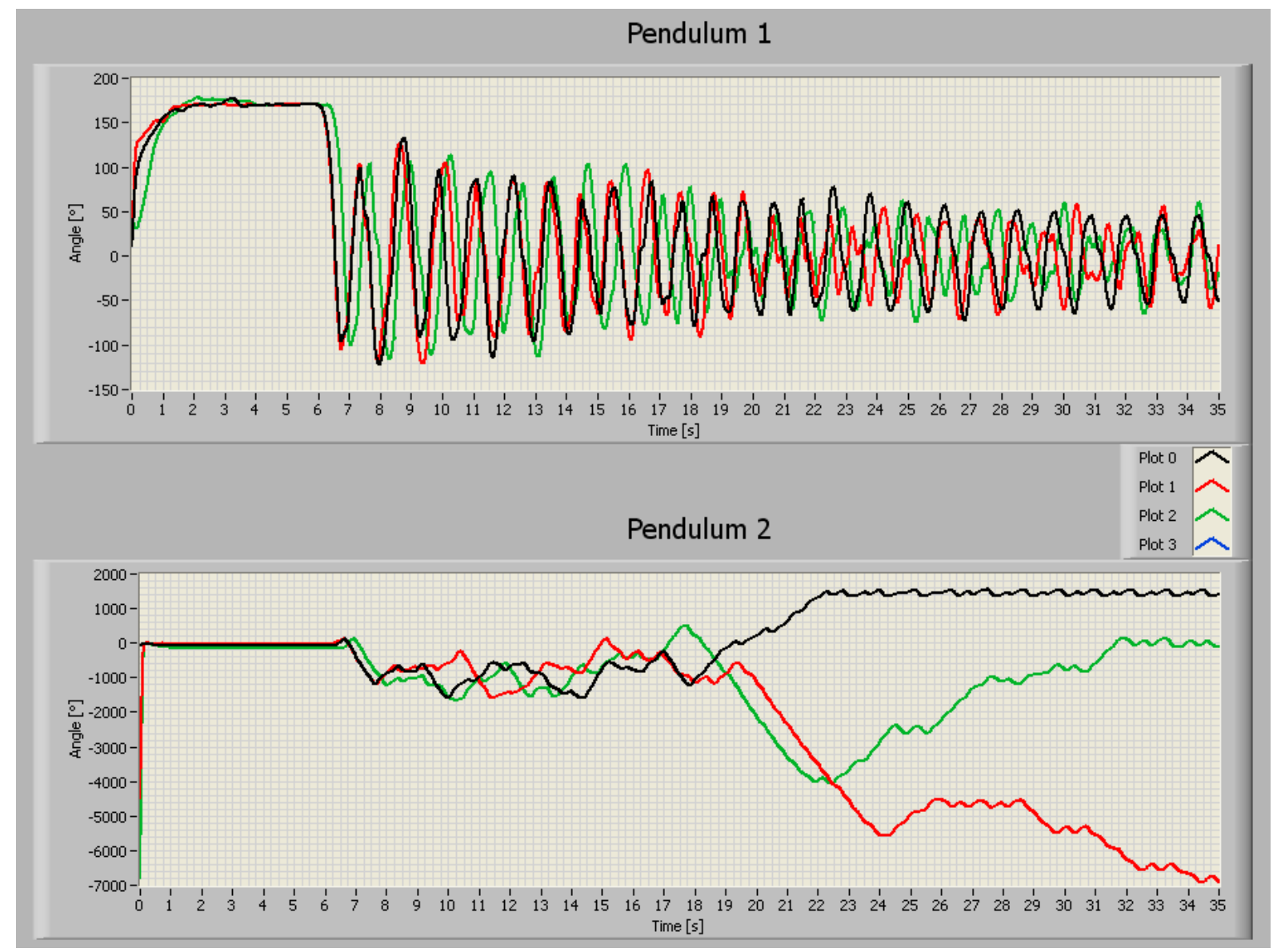

Figure 6. Demonstrating the dependence on initial conditions on-line with plotting angular displacements of different movements that started from nearly identical coordinates (marked with different colours). The values of the angular displacement of the lower pendulum are untruncated, so the angles beyond and under 360 degrees mark pendulum flips.

\subsection{Phase portraits and phase space section}

The software can display the phase portraits of the two pendula real-time (i.e. the angular velocity as a function of angular displacement, shown in Figure 7). Besides that, it is able to calculate a phase space section, and display the new trajectory intersections real-time (shown in Figure 7). The examined phase space section:

$$
\omega_{2}-\theta_{2} \text { plane, when } \theta_{1}=0 \text { and } \omega_{1}>0
$$

This is a practical choice, because the upper pendulum that never (or rarely) flips and passes through the vertical state $\left(\theta_{l}=0\right)$, and the angular displacement of the lower pendulum (which flips many times) can be examined in the whole range. 


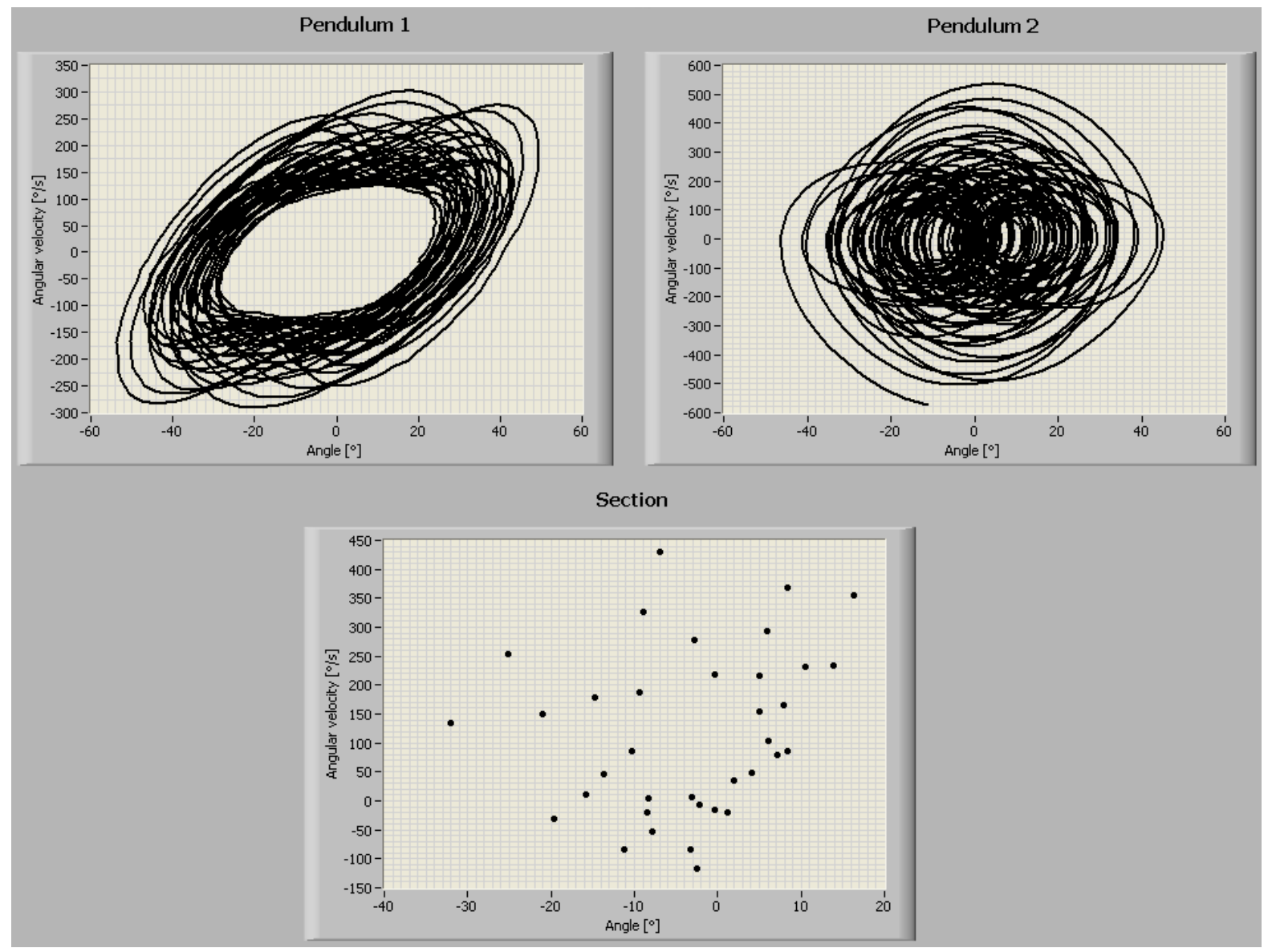

Figure 7. Online demonstration of the analysis techniques of chaotic motion. The upper panels show the phase portraits of the two pendula (with 'Pendulum 1' denoting the upper and'Pendulum 2' the lower one) whilst the lower panel depicts a phase space section with the parameters shown in the text.

Displaying these sections helps to monitor the chaotic motions in real-time and it demonstrates some typical analysis techniques (such as how to use them in a parametric identification of the motion of the double pendulum motion [17]). We can compare the phase space sections of different movements that started from nearly identical coordinates offline more precisely, and it is an instructive exercise for undergraduate students.

\section{Offline analysis}

Although the software presented above is able to compare data of different movements on-line to a certain extent, to calculate the characteristic numbers and sections we need an accurate synchronisation of the data of different measurements, so another software was made for precise offline analysis. The software displays the elements of the phase space $\left(\theta_{1}, \theta_{2}, \omega_{1}, \omega_{2}\right)$ with different colours for different measurement data, so the synchronisation can be done manually (as shown in Figure 8.). 


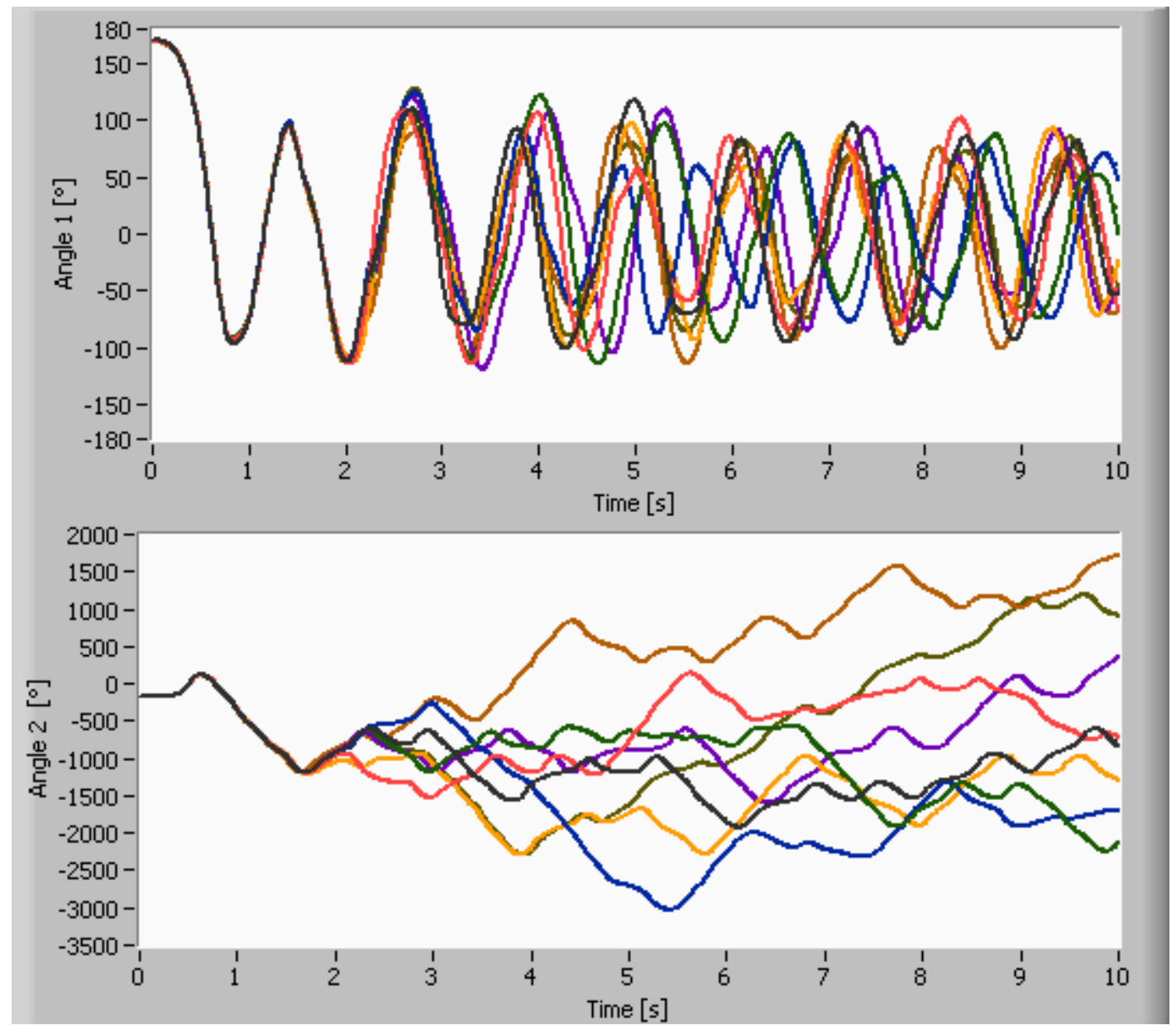

Figure 8. Off-line comparison of different measurements started nearly identical initial conditions by plotting the synchronised angular displacements (with 'Angle 1' denoting angular displacement of the upper pendulum and 'Angle 2' that of the lower one) with different colours.

In the experiment 8 trajectories were measured, with the motions launched from the most similar initial conditions possible. Figure 8 shows the movements are almost identical before the first unstable state, where they separate. The offline software can compare either pair of the motions and display the time development of their distance in the phase space.

The initial conditions were (with $0.8^{\circ}$ resolution):

$$
\theta_{1}=169.5^{\circ}, \omega_{1}=0 \%, \theta_{2}=-179.5^{\circ}, \omega_{2}=0 \%
$$

\subsection{Lyapunov exponent}

The coordinate space of the frictionless double pendulum can be divided to different domains as the initial values of the coordinates determine the nature of the motion (periodic, quasi-periodic or chaotic) and their maximum Lyapunov exponents. However, the friction in the real experiment changes the nature of the motion and the values of the exponents during the motion.

By fitting an exponential curve on the whole chaotic part after the separation of two paths, the global Lyapunov exponent can be calculated; however, to define the accurate length of the chaotic part of the motion is generally difficult. Determining the local Lyapunov exponent (fitting to a short time period in this case) allows us to compare the measurements with numerical simulations. Shinbrot et al [9] examined the short period immediately after the beginning of the motion and compared them to theoretical results calculated for the energy level determined by the initial conditions. Calculating the distance in the phase space of the compared trajectory pairs and fitting the exponential were done for the time interval for which the reduced $\chi^{2}$ was minimum (see details in [9]). However, they mentioned 
that to use a fixed, short time interval is suitable in a demonstration experiment because the exponent will be a positive number and will show the chaotic behaviour too.

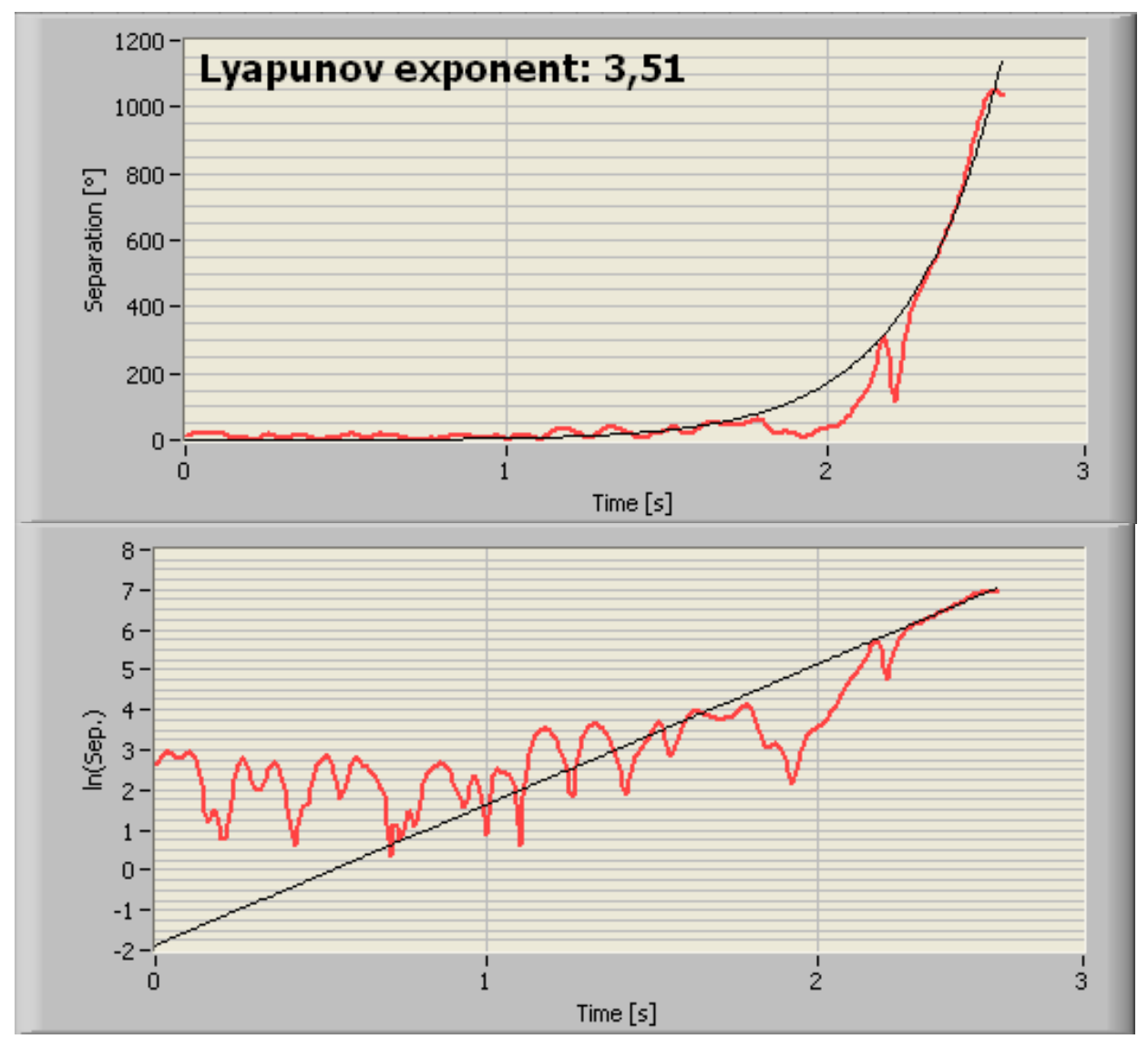

Figure 9. Fitting an exponential to the distance in phase space of two trajectories (black and blue curve in Figure 8.), that started with nearly identical initial conditions (shown above). The length of the examined time interval was enumerated with a reduced $\chi^{2}$ method [9], and the local Lyapunov exponent determined is shown on the top. The graph at the bottom plots the linearized version of the top curve, and it shows the problem of the method and the importance of the unstable states of the double pendulum much better, as it is discussed in the text.

The local Lyapunov exponents that were determined with the method proposed by Shinbrot et al [9] were positive for every trajectory pair in our measurements (an example is shown in Figure 9.), therefore chaotic behaviour of the examined real double pendulum is demonstrated in this starting region. However, the separation plotted in logarithmic scale (bottom graph in Figure 9.) shows that the exponential growth is not a prefect approximation, but also, comparing with the evolution of $\theta_{1}, \theta_{2}, \omega_{1}, \omega_{2}$, we can see that local minimums of the curves are marking the unstable states of the double pendulum. Thus, to guess the length of the time interval to calculate the local exponents, we propose to consider the time elapsed between two unstable states of either of the pendula. These states divide the movements into parts that seem to be instructive to enumerate the local exponents and to compare with theoretical results.

\subsection{Phase space section}

In conservative case, the movement of the double pendulum gives a typical Poincare section that is different in every energy level. With increasing values of the kinetic energy, the trajectories from the ordered picture of low-energy movements are disintegrating to chaotic regions. However, the motion is unpredictable, we know places in the section called 'regular islands', where the trajectories will not cross over, making these islands the characteristic elements of the sections $[1,6]$.

As we see in Section 5.1, in dissipative case, the friction blurs the chaotic, quasi-periodic and periodic domains. Therefore, if we create a phase space section using the calculation method of a Poincaré 
section, which assumes constant energy-level (that is why $\omega_{1}$ could be not a fixed value), a short time interval of the trajectories should be examined to compare with the theoretical sections of the actual kinetic energy values.

The other problem is that a phase space section of one experiment consists only of a few points, a whole movement of the real double pendulum is not enough long to plot a section like a nice Poincaré section (as it mentioned in Conclusion of Ref [18]).

So, analyzing the evolution and the structure of intersections of the different trajectories started with nearly identical initial conditions in one plot is an exciting exercise, and one can use it to compare the measured movements with theoretical Poincare sections or with data obtained with numerical simulations taking the dissipative components into account.

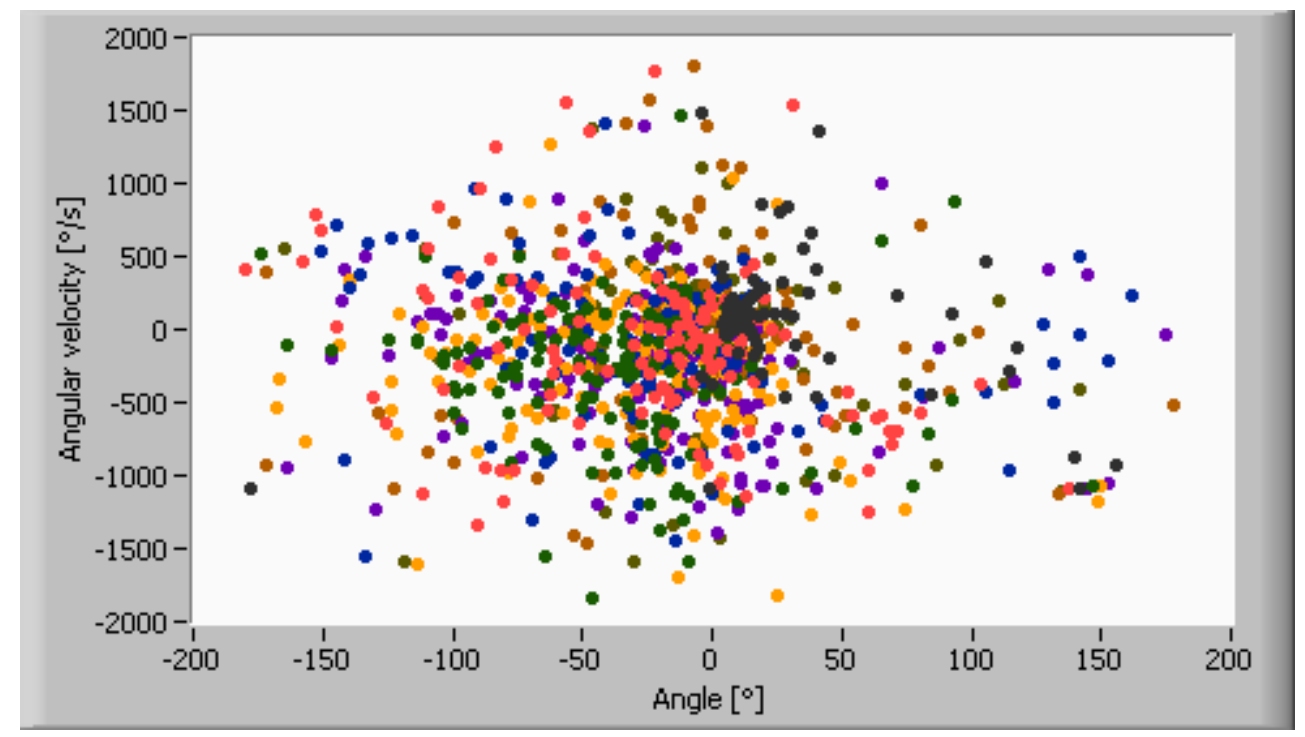

Figure 10. A phase space section with 8 movements started with nearly identical initial conditions plotted with different colours. $t=55 \mathrm{~s}), \omega_{2}-\theta_{2}$ plane, when $\theta_{1}=0$ and $\omega_{1}>0$.

Our phase space section (Figure 10.), which was made as described above, were calculated using the first 55 seconds of the motion, which is a considerably longer time that is needed for the comparison with numerical models, but it demonstrates the evolution and the structure of the intersections.

We can see that at close to the end of the motion the intersection points are converging to the origin of the section, furthermore it is revealing that the intersections are not concentrated in typical sections and the structures are completely different.

\section{Conclusions}

As reviewed above, the measurement system presented here is able to demonstrate the main characteristics of chaos and its study methods in the eye-catching movement of the real double pendulum.

The simple set-up of the measurement system - without an expensive and complicated hardware background - makes the methods widely accessible. Using and comparing the different measurement theories and numerical methods helps to teach the ways of problem solving and different measurement methods.

Using the system is very simple, only attaching a Bluetooth USB dongle to the PC is needed. The presented open-source LabVIEW software (which is downloadable from [19], where are our system set-up, parameters, screenshots and measurement results are presented) can easily be changed to allow further expansions, for example, to develop the experiment to a Remote Laboratory experiment.

The software is able to display the parameters, characteristic chaotic numbers and sections of the spectacular motion in real time. The presentation of the sensitive dependence on initial conditions and the display of the phase portraits and phase space sections qualifies the system as a demonstration tool in high-school education. Furthermore, the analysis of the measured data is an instructive exercise for the undergraduate physics students studying the analysis of mechanical and chaotic systems. 
As our results show, the friction affects the examined motion radically, so using the analysis methods shown above and comparing the measured movement with data obtained with numerical simulations taking the dissipative components into account could be very instructive. Measuring the Lyapunov exponents and analysing the evolution of the phase space sections and the berth of its trajectory intersections on the appropriate theoretical Poincaré sections could offer new results and can prove to be an exciting exercise for undergraduate students.

\section{Acknowledgments}

We thank Iván Gyémánt (Department of Theoretical Physics, University of Szeged) for his useful comments in the analysis of the chaotic motion and Péter Makra (Department of Medical Physics and Informatics, University of Szeged) for revising the manuscript. We also thank Imre Szabó (Department of Experimental Physics, University of Szeged) for preparing the double pendulum examined. This work was supported by TÁMOP-4.2.1/B-09/1/KONV-2010-0005.

\section{References}

[1] Tél T and Gruiz M 2006 Chaotic Dynamics (Cambridge: University Press Cambridge)

[2] Adams H M and Russ J C 1992 Chaos in the classroom: exposing gifted elementary school children to chaos and fractals J. Sci. Edn Technol. 1 191-209

[3] Bae S 2009 Chaos: a topic for interdisciplinary education in physics Eur. J. Phys. 30677

[4] Vankó P 2007 Investigation of a chaotic double pendulum in the Basic Level Physics Teaching Laboratory Eur. J. Phys. 28

[5] University of Florida, Department of Physics, PHY4803L Advanced Physics Laboratory http://www.phys.ufl.edu/courses/phy4803L/group_IV/chaos/Chaos.pdf

[6] Hand L N and Finch J D 1998 Analytical Mechanincs Cambridge: University Press Cambridge)

[7] Stachowiak T and Okada T 2006 A numerical analysis of chaos in the double pendulum Chaos, Solitons and Fractals 29 417-422

[8] Bender C M, Feinberg J, Hook D W and Weir D J 2009 Chaotic systems in complex phase space Pramana J. Phys. 73 453-470

[9] Shinbrot T, Grebogi C, Wisdom J, and Yorke J 1992 A Chaos in a double pendulum American Journal of Physics $\mathbf{6 0} 491$

[10] Suzuki M, Toho M, Minato A and Ozawa S 2001 Computer Aided Education in Physics by using Double pendulum Equipment International. Conference Information. Technology Based High Education and Training, Kumamoto http://www.eecs.kumamoto-u.ac.jp/ITHET01/proc/036.pdf

[11] Lawrence P, Stuart M, Brown R, Tucker W and Sainudiin R 2010 A Mechatronically Measurable Double Pendulum for Machine Interval Experiments http://www.math.canterbury.ac.nz/ r.sainudiin/preprints/MeasurableDoublePendulum.pdf

[12] Marone M 2002 The Mercer online interactive chaotic pendulum Computing in Science and Engineering 4 94-97

[13] Freescale Semiconductor, Inc. - MMA7360L 3-axis acceleromater http://www.freescale.com/files/sensors/doc/data_sheet/MMA7360L.pdf?fsrch $=1$

[14] Freescale Semiconductor, Inc. - MMA73x0L Analog Output Accelerometer Evaluation Board http://cache.freescale.com/files/sensors/doc/app_note/AN3376.pdf

[15] Avago Technologies - AEDB-9140 Series Three Channel Optical Incremental Encoder Module with Codewheel - http://www.avagotech.com/docs/AV02-1584EN

[16] Rayson Technology Co., Ltd. - Bluetooth Class 2 CSR BC4-ext module http://www.cs.put.poznan.pl/wswitala/download/pdf/btm112_datasheet.pdf

[17] Liang Y, Feeny B F 2008 Parametric identification of a chaotic base-excited double pendulum experiment Nonlinear Dyn 52 181-197

[18] Levien R B and Tan S M 1993 Double pendulum: An experiment in chaos American Journal of Physics $\mathbf{6 1} 1038$

[19] Homepage of the presented double pendulum and measurement system http://www.inf.u-szeged.hu/noise/Research/DoublePendulum/ 